\title{
Computer Science in the Management of Operating Theatres: A Challenge for Sub-Saharan Anaesthetists and Resuscitation Medical Doctors
}

\author{
M. Diedhiou ${ }^{* 1}$, JN. Tendeng ${ }^{2}$, D. Barboza ${ }^{3}$, A.K. Diallo ${ }^{3}$, ML. Diao ${ }^{2}$, G. Camara ${ }^{3}$, I. Konate ${ }^{2}$ \\ ${ }^{1}$ Anaesthesia, Resuscitation Department, Saint Louis Regional and Teaching Hospital /Senegal, \\ Gaston Berger University (UGB) Saint Louis \\ ${ }^{2}$ General Surgery Department Saint Louis hospital, Gaston Berger Saint Louis University \\ ${ }^{3}$ Department of Applied Science and Technology Faculty/ Gaston Berger University (UGB) Saint Louis
}

*Corresponding Author: M. Diedhiou; moustapha.diedhiou @ ugb.edu.sn

Received 22 April 2020;

Accepted 18 May 2020;

Published 01 June 2020

\begin{abstract}
Introduction: Anesthetists and resuscitation doctors often coordinate the management of operating theaters. Information systems that are an importants tools for operating theatre management are not commonly used in Senegal. The objective of this study is to evaluate the contribution of computer sciences in improving the management and quality of care in the operating theatre. Material and Method: Prospective evaluation over one year of the quality of the standardized discharge summaries (anaesthetic and surgical protocols) provided by the SIMENS software based on the analysis of a Hospital Information System (HIS) use indicators: comprehensiveness, completeness, and validity. Results: The overall comprehensiveness on the use of the Information System (IS) in the Operative Room (OR) was 89\%, the completeness of the fields was optimal in $96.6 \%$ of the cases for the operating protocols and in $80 \%$ of the cases for the anesthetic protocols. $64 \%$ of the anesthetic protocols were considered valid compared to $73.3 \%$ validity for the surgical protocols. Discussion/Conclusion: The computerization of operating theatres is a long-term process and will ultimately improve the quality of care by having an impact on quality management. Improving the indicators of practical use of an IS in the operating theatre (comprehensiveness, completeness and validity of RSS) requires good involvement of all actors of the theatre environment. The benefits of computerization would be more relevant once the services associated with the operating theatre (hospital services, stretcher-boarding, etc.) are connected to the computer system, but also by automating the input of information to be included in anaesthetic and surgical protocols.
\end{abstract}

Keywords: Hospital information system, computerization of the operating theatre, organization of the operating theatre, anaesthetist doctor

\section{Introduction}

The management of operating theatres is often entrusted to the anaesthetists. They have an almost daily presence in the operating theatre independently of the elective cases. They interact with almost all the actors of the operating theatre, giving them a crosssectional view of the patient and the operating environment.

For decades, new information and communication technologies (NICTs) have become part of our daily lives and have revolutionized improvements in the medical field (MRI, laparoscopic surgery, radiofrequency, e-health, telemedicine, etc.) [1]. Operating theatre computerization systems, like hospital information systems (HIS), are nowadays an essential tool for improving the quality of care in this environment where organization and management are a pillar due to the diversity of stakeholders, actors and procedures.

The use of information systems in the operating theatre is nevertheless not common practice in Senegal. At the Saint Louis hospital center, in the context of diversification of anaesthetic and operative acts and an increase in the number of operating theatre staff, the project to computerize the operating theatre using SIMENS software (Senegal's National Medical Computerization System) was set up in collaboration with the computer unit of the Gaston Berger University in the region.

The authors of this work propose to evaluate the contribution of the SIMENS software in improving the management and quality of care in the operating theatre.

\section{Material and method}

This is a prospective study, extending over one year (August 1st, 2016 to August 1st 2017) conducted in the central operating theatre of the regional hospital of Saint Louis in Senegal. In August 2016, the anesthesiology and intensive care department in collaboration with the IT department of the University Gaston Berger initiated the project of computerization of the central operating theatre of the regional hospital of Saint louis in Senegal. We then proceeded 
with the computerization of the operating theatre planning, the surgical and anaesthetic protocols previously written manually in a register. All the computerized records of patients admitted to the operating room since August 1st 2016, for all surgeries, were included in our study. In practice, we based our work on the one hand on the analysis of general statistical data extracted from the information system and on the other hand on the evaluation of the quality of the standardized discharge summaries, in this case the surgical and anaesthetic protocols provided by the abovementioned system.

We thus carried out a random selection of 50 surgical and anaesthetic protocols per month over the whole study year, i.e. a total of 600 surgical protocols and 600 anaesthetic protocols. The evaluation of the quality of the protocols provided is based on the process of prioritization, selection and extraction of the relevant data to be included in an operating or anaesthetic protocol (civil status data, safety checklist, diagnoses, procedures, type of anaesthesia, complications, etc.). The verification procedure covered all the variables contained in the surgical and anaesthetic protocols. Compliance was assessed according to the following criteria: comprehensiveness (the percentage of patients admitted to the block with a computerized record), completeness (the percentage of the number of fields filled in compared to the total number of fields on the protocols), validity (the final obtaining of protocols meeting the standards). An operating protocol is considered valid if it meets the standards previously established by the surgical team, i.e. a protocol where the six fields (position, type of anaesthesia, exploration approach, procedures, incidents, postoperative care) are correctly filled in. In the same way, an anaesthetic report is considered valid if it meets the standards previously established by the anaesthetists: i.e. a good filling of all the fields which are safety check list, IV line and monitoring, installation, pre-filling, induction, airway control mode, maintenance, intraoperative haemodynamic status, water balance, anaesthetic incidents, mode of awakening and/or extubation.

\section{Results}

During the period of the study 1590 patients were anaesthetized in the operating room of the Saint Louis Regional Hospital and only 1413 patients with a computerized record were collected in our study, i.e. a comprehensiveness rate of $89 \%$. Only 2 departments (in this case the department of pediatric surgery and urology) out of a total of 7 departments had a comprehensiveness rate of $100 \%$. The comprehensiveness rate was $52 \%$ in the first quarter of our study, $72 \%$ in the second, $55 \%$ in the third $87 \%$ and $90 \%$ in the fourth. The distribution of the number of patients per service was $25.6 \%$ for general surgery; $23.4 \%$ for paediatric surgery; $21.2 \%$ for orthopedic surgery... Figure 1 shows the distribution of the number of patients by department and the sex ratio of our population.

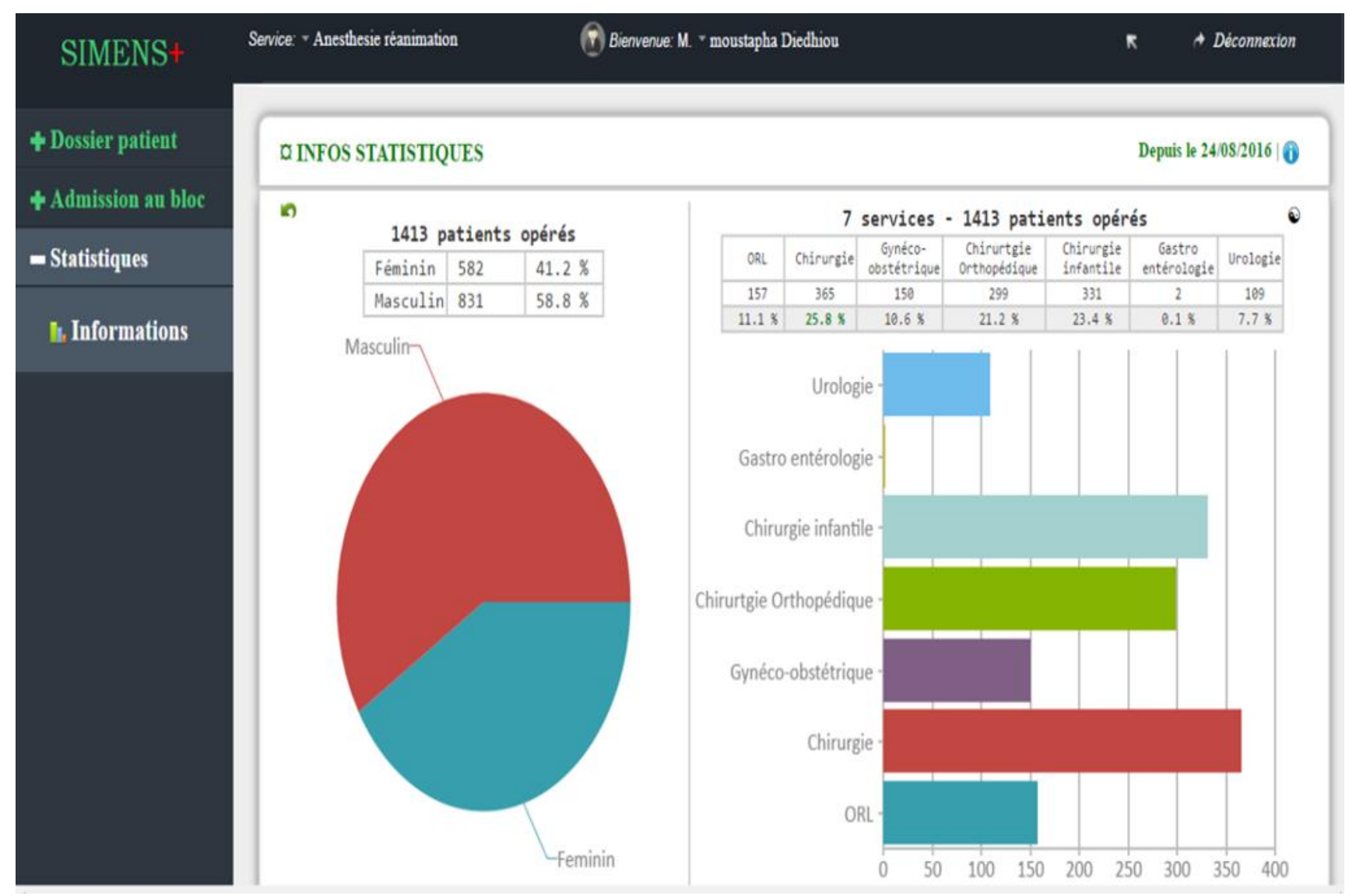

Figure 1: General statistical data provided by SIMENS software

\section{$>$ Results of the quality assessment of the operating protocols:}

In our randomized sample of 600 patients, the completeness rate was $100 \%$ in 582 patients, $85.7 \%$ in 15 patients and $57.1 \%$ in 3 patients. Of the 600 randomly selected surgical protocols, 440 protocols or $73.3 \%$ were considered valid and 160 or $26.6 \%$ were not.
Table 1: Completeness of operating protocols

\begin{tabular}{|c|c|c|}
\hline $\begin{array}{c}\text { Completeness } \\
\text { rate }\end{array}$ & $\begin{array}{c}\text { Number of fields } \\
\text { filled / 7 fields }\end{array}$ & $\begin{array}{c}\text { Number of patients } \\
(\%)\end{array}$ \\
\hline $57,1 \%$ & 4 & $3(0,83 \%)$ \\
\hline $85,7 \%$ & 6 & $15(2,50 \%)$ \\
\hline $100 \%$ & 7 & $582(96,67 \%)$ \\
\hline Total & - & $600(100 \%)$ \\
\hline
\end{tabular}


Table 2: Validity of protocols

\begin{tabular}{|l|l|}
\hline Status of operating protocols & Number of protocols (\%) \\
\hline Invalid & $160(26,67 \%)$ \\
\hline Valid & $440(73,33 \%)$ \\
\hline Total & $600(100 \%)$ \\
\hline
\end{tabular}

Results of quality assessment of anaesthetic protocols: In our sample of 600 patients, the rate of completeness of anaesthetic protocols was $100 \%$ in 480 patients, $72 \%$ in 82 patients and $58 \%$ in 38 patients.

Out of the 600 anaesthetics selected at random, 440 protocols or $73.3 \%$ were considered valid and 160 or $26.6 \%$ were invalid.

Table 3: Completeness of anaesthetic protocols

\begin{tabular}{|c|c|c|}
\hline $\begin{array}{c}\text { Completeness } \\
\text { rate }\end{array}$ & $\begin{array}{c}\text { Number of fields } \\
\text { filled / 12 fields }\end{array}$ & $\begin{array}{c}\text { Number of patients } \\
(\%)\end{array}$ \\
\hline $58 \%$ & $7 / 12$ & 38 \\
\hline $72 \%$ & $9 / 12$ & 82 \\
\hline $100 \%$ & $12 / 12$ & $480(80 \%)$ \\
\hline Total & - & $600(100 \%)$ \\
\hline
\end{tabular}

Table 4: Validity of anaesthetic protocols

\begin{tabular}{|l|l|}
\hline Status of anaesthetic protocols & Number of protocols (\%) \\
\hline Invalid & $215(36 \%)$ \\
\hline Valid & $385(64 \%)$ \\
\hline Total & $600(100 \%)$ \\
\hline
\end{tabular}

\section{Discussion}

In a hospital facility, the operating theatre represents one of the major sectors and certainly one of the most emblematic, as the centerpiece of the technical platform ${ }^{[2]}$. It is a place where highly technical procedures are carried out, which often represent the facility's "flagship activities", but whose cost and financial investment necessarily imply notions of profitability to which are added unavoidable safety imperatives. The diversity of the procedures performed, the fact that they are carried out in a regulated activity or in an emergency, the cohabitation between different specialties, the multiplicity of human resources involved, the need to comply with regulations and the constant concern for quality are all elements to be taken into account in the proper management of an operating theatre ${ }^{[2]}$. The organization of such a structure is therefore a real technical but also organizational challenge.

Besides, isn't it the dream of every manager to have a transversal and permanent vision on the activity of his company? Wouldn't it be desirable for a manager to claim omnipresence in order to better understand all aspects of his structure? Here are as many ideals that anaesthetists and operating theatre coordinators would undoubtedly like to achieve. The computer tool, by the control of the data it brings, by the facilitation of the evaluation work it allows, is one of the solutions to take up this challenge. Operating theatre computerization systems are tools for achieving these objectives, which are part of the principles of quality management. The hospital in Saint Louis has a bedding capacity of 240 beds. All surgical specialties (except neurosurgery and cardiovascular surgery) exist there and flourish in an operating theatre with 4 operating rooms where an average of 40 anaesthetic procedures are performed per week. The nursing staff of the operating theatre is composed of one nurse anaesthetist and two nurse-instrumentalist per operating theatre; they work under the respective responsibility of two intensive care anaesthetists and nine surgeons of all specialties.

In this context of diversification and increase of anaesthetic procedures, it was then imperative to develop a strategy of improvement in the organization of the operating theatre. Thus, the anaesthesia department in collaboration with the computer department of the University Gaston Berger has introduced the use of SIMENS software (Senegal's national medical computerization system) in the operating theatre. SIMENS is a Hospital Information System (HIS) developed and designed by the computer department of the Gaston Berger University. This system was modelled according to the needs previously estimated by the hospital's medical teams. The computerization of an anaesthesia department concerns three areas: the patient record (anaesthesia sheet, anaesthesia consultation, prescription), the planning (operating theatre, consultation) and the office automation of the department (bibliography and especially the doctor's diary, nursing planning, archives of anaesthesia records and procedures) ${ }^{[1]}$. A computerization project in the operating theatre is a long-term project, spread over several years, in successive stages ${ }^{[1]}$. The implementation is complicated and it is strongly recommended that it be divided into several sub-projects that will be easier to complete $^{[1]}$. The computerization of the operating theatre in Saint Louis via SIMENS is part of this ambition of progressive evolution and constitutes the initial phase of the major project to set up an HIS throughout the hospital. In addition, the national hospital expertise and audit mission reminds us that in an operating theatre $<$ the computer input of all the data relating to the path of the operated patient will constitute an indispensable working base in the concern for an approach of improvement and optimization of the means ${ }^{[3]}$; the ideal is to have an adapted computer tool in order to integrate all these data, with a recording of the various time parameters in particular ${ }^{[4]}$. Improving the quality of care in the operating theatre therefore certainly involves making good use of the information system, but also optimizing the indicators of practical use of the computer tool: comprehensiveness, completeness, validity (5), etc. ${ }^{[6]}$. According to JL Bourgain and colleagues, comprehensiveness is one of the first and most important questions to ask before using an HIS database for statistical purposes. Comprehensiveness is easy to measure for data that have no possibility of being null and void, such as duration (anesthesia or surgery), operators. It cannot be assessed for data such as drug or medical device use because it is not possible to differentiate a null value (the drug was not injected) from missing data. Comprehensiveness increases over time and after five years of use, missing data are in the order of 2 to $5 \%{ }^{[1]}$. Saada Chougrani et al in their study on the evaluation of the hospital information system of the Oran University Hospital found a total comprehensiveness rate of $59.2 \%$ and completeness for medical data relating to follow-up of $50 \%{ }^{[5]}$. In our study the overall comprehensiveness was $89 \%$ with a clear progression over the months and completeness was $80 \%$ for anaesthetic protocols and $96.6 \%$ for surgical protocols. These differences in comprehensiveness and completeness rates can be explained by the fact that the Oran study concerned the evaluation of the information system in the whole hospital, whereas in our study the evaluation only concerned the information system in the OR, with a better control and communication with users in this limited environment. In the Oran study, the overall validity of the standardized discharge summaries produced at the facility level was $60.5 \%$, varying, depending on the associations, from $28.6 \%$ (lung-thorax) to $84.6 \%$ (mother-child). In our study, the validity of the surgical and anaesthetic protocols in our sample was $73.3 \%$ and 
$64 \%$ respectively. This variability of validity rates in the Oran study and in our study reflects the impact of the human factor in any computerization process; and that the perfection of an information system can only be achieved through the unavoidable adhesion of all the actors, even if some authors such as Devitt JH and colleagues ${ }^{[6]}$ recommend that the computerization of the data input of information that must appear on a surgical or an anaesthesia protocol allows to improve the validity, comprehensiveness and completeness of the standardized output summaries. These concepts also require that all monitoring devices, anaesthesia ventilators, etc. be connected to the Information System. Moreover, one of the limits of our computer tool remains the lack of connectivity with the ancillary services in the operating theatre but this certainly does not reduce the relevance of our information system because $<$ The quality of the anaesthesia report has a potential influence on the vital prognosis of patients and certainly on the quality assurance strategy $>>{ }^{[7]}$

The effectiveness of an information system on the functioning of the operating theatre depends on connectivity with these ancillary services. This vision is shared by A champault and colleagues who defend that $\ll$ the operating theatre manager, assisted by the operating theatre board, can propose a certain number of improvements and correct the most obvious dysfunctions, while knowing that this is not as simple and that wanting to reduce the time between two surgeries, for example, can run up against many difficulties. $>>{ }^{[8]}$. In our work, the absence of taking deadlines into account is not an omission but rather fits in with the principle of progressive evolution of the computerization process, which is conceived with greater speed and more ownership with an already experienced team.

\section{Conclusion}

The computerization of the operating theatre is a long-term process and ultimately improves the quality of care by having an impact on quality management. The implementation of an information system in the operating theatres of a developing country like Senegal is a godsend for the improvement of management in this emblematic sector of hospitals. However, the real impact of an information system in the operating theatre would be better perceived when it is integrated into a large hospital information system.

\section{Ethics approval and consent to participate}

Not applicable

\section{Abbreviations:}

SIMENS: Senegal's National Medical Computerization System HIS: Hospital Information System

SI: Computerization system

RSS: Standardized discharge summaries

UGB: Gaston Berger University

UCAD: Cheikh Anta Diop University of Dakar
The data are available on SIMENS (Senegal's National Medical Computerization System)

\section{Conflict of interest}

The authors do not declare any conflict of interest.

\section{Funding Statement}

The researches and the publication were done by self-funding.

\section{Authors' contributions}

'MD, JNT, DB and MLD were in charge of the collection, analyzation and interpretation of the data. GC helped with the use of the software and statistics calculations. All authors read and approved the final manuscript',

\section{Acknowledgments}

Dr T.Y-E.A DONYO for helping translating the manuscript in English

Pr Ibrahima Konate for his enlightened leadership

\section{References}

[1] The computerization of an operating theatre JL Bourgain, M Buset, JM Puizillout, JM Ropars - Update conferences. FSSR, 2006

[2] Organization and management of operating theatres $G$ Adda - Hygiene and safety in health care institutions, ..., 2002

[3] MeaH (National hospital expertise and audit mission). Management and organization of operating theatres. 2006.:

http://www.meah.sante.gouv.fr/meah/index.php?id=1147

[4] Computer tools in the management of the operating theatre P Beutter - Revue hospitalière de France, 1999 kki

[5] Evaluation of the hospital information system of the Oran University Hospital facility S Chougrani, S Ouhadj, F Agag - Public health, 2013 - cairn.info

[6] Devitt JH, Rapanos T, Kurrek M, et al. The anesthetic record: accuracy and completeness. Can J Anaesth 1999; 46: $122-8$.

[7] Sanborn KV, Castro J, Kuroda M, et al. Detection of intraoperative incidents by electronic scanning of computerized anesthesia records. Comparison with voluntary reporting. Anesthesiology 1996; 85: 977-87.

[8] Can we reduce the time between two surgeries in the operating room? Prospective study A Champault, V Arsena, C Barrat, P Bayeh - Annales de chirurgie, 2003 Elsevier

\section{Data Availability}

